\title{
La voix et le souffle chez John Marston
}

\section{Marc Bonini}

\section{(2) OpenEdition \\ Journals}

Édition électronique

URL : http://journals.openedition.org/shakespeare/337

DOI : 10.4000/shakespeare.337

ISSN : 2271-6424

\section{Éditeur}

Société Française Shakespeare

\section{Édition imprimée}

Date de publication : 1 novembre 1999

Pagination : 63-74

ISBN : 2-84269-331-0

\section{Référence électronique}

Marc Bonini, «La voix et le souffle chez John Marston », Actes des congrès de la Société française Shakespeare [En ligne], 17| 1999, mis en ligne le 01 novembre 2007, consulté le 01 mai 2019. URL : http://journals.openedition.org/shakespeare/337 ; DOI : 10.4000/shakespeare.337 


\section{S H A K E S P E A R E \\ \& $\quad$ L $A \quad$ V O I X}

Société Française Shakespeare

Actes du Congrès de 1999

米米

Textes réunis et présentés par

Patricia DORVAL

publiés sous la direction de

Jean-Marie MAGUIN 


\section{LA VOIX ET LE SOUFFLE CHEZ JOHN MARSTON}

«Comedies are writ to be spoken, not read : remember the life of these things consists in action»: nous tenons de Marston luimême, dans sa préface à Parasitaster, or the Fawne, ce jugement sur 1 'importance du jeu d'acteur et de la voix. De fait, on trouve dans ses pièces tout un arsenal de techniques qui mettent en valeur les possibilités de la voix : scènes d'écho, onomatopées, madrigalismes dans les dialogues... les exemples ne manquent pas. Notre préoccupation, cependant, est plus théorique : il s'agit d'analyser, dans le dyptique formé par Antonio and Mellida et Antonio's Revenge, les rapports qu'entretiennent la voix et le souffle. Cette équivalence bien attestée en anglais élisabéthain entre «voice» et «breath», Marston la thématise assez longuement dans ces pièces expérimentales où le discours métadramatique sur la voix de l'acteur affleure presque à chaque ligne.

Comprendre la voix comme souffle, c'est en apparence l'ancrer fermement dans un corps auquel elle appartiendrait comme les artères ou le sang. Telle est la signification de la théorie répandue dans l'ancien stoïcisme qui définit l'émission vocale comme $\pi v \varepsilon v \mu \alpha$, c'est-à-dire comme un souffle chaud, connaturel au corps, qui passe par la bouche et produit des vibrations dans l'air. Chez Marston, en revanche, et particulièrement dans les pièces du début de sa carrière, il semble que la voix ne prenne pas son origine dans le corps mais à l'extérieur de lui, dans une force puissante et indépendante, un souffle qui devient voix lorsqu'il est porté par le corps. 
Dans nos analyses sur le souffle, nous essaierons donc de prolonger le concept de «dislocation» que Jonathan Dollimore a exploré avec pertinence dans ces pièces, essentiellement sur le plan de la personnalité et de l'idéologie ${ }^{1}$. Le phénomène connexe qui nous intéresse ici, c'est une sorte de dislocation vocale, tension douloureuse entre le désir de s'appartenir pleinement, de contrôler sa propre voix, et une prise de conscience non moins forte que cette puissance qui nous traverse est liée à une force supérieure incontrôlable. Nous étudierons surtout deux types de voix dans le conflit politique qui sous-tend les deux parties d'Antonio and Mellida: celle du tyran, Piero, et celle de son jeune adversaire Antonio. En contrepoint à ces deux voix dissociées que Marston nous présente, le texte comporte également le personnage de Balurdo, dont les prétensions et les facéties imitent en les grossissant les traits vocaux et les aspirations des deux autres personnages.

\section{L'impérialisme vocal de Piero}

L'arrivée spectaculaire de Piero, duc de Venise, dans la première scène d'Antonio and Mellida donne lieu à une confrontation entre le tyran et l'un de ses opposants, Felice. Au moment précis où Piero annonce d'une voix tonitruante le résultat de ses exploits militaires, un signe acoustique complexe (le bruit d'un tremblement de terre) vient à la fois ponctuer et commenter cette prise de parole :

Piero. Victorious fortune, with triumphing hand,

Hurleth my glory 'bout this ball of earth,

Whilst the Venetian duke is heavèd up

On wings of fair success, to overlook

The low-cast ruins of his enemies,

To see myself adored and Genoa quake :

My fate is firmer than mischance can shake.

Felice. Stand! The ground trembleth.

Piero. Ha, an earthquake!

Balurdo.

O, I smell a sound. ${ }^{2}$

Balurdo, spécialiste ès synesthésies en tous genres, apporte ici, de façon typiquement marstonienne, juste une pointe de grotesque pour ponctuer un échange sérieux. C'est le même Balurdo qui plus tard, dans Antonio's Revenge, demandera à l'assistance après quelques 
sons pincés à la viole de gambe : «did you ever smell a more sweet sound ?» (III.3.23-4). Dans un premier sens, le bruit du tremblement de terre fonctionne comme une illustration sonore des mots «quake» et «shake» accentués par Piero, comme si Marston avait voulu par ce signal acoustique représenter la plainte de Gènes défaite par la flotte vénitienne. Mais ce signe est autrement plus complexe, et donne bientôt à Felice l'occasion d'un commentaire politique sur la voix du souverain et sa véritable source.

Dès les premières lignes du discours de Piero, nous remarquons que le tyran se décrit comme étant sous l'emprise d'une force supérieure (la fortune) qui le guide et le manœuvre à sa guise : l'auteur de l'action est «victorious Fortune» et non «victorious Piero»; le duc se déclare passif, il ne fait que constater son destin («see myself adored»). Affirmation du pouvoir et en même temps retrait au nom d'une puissance plus grande : telle est selon Michel Foucault la caractéristique du discours d'autorité, dont la voix se déguise pour mieux asseoir sa domination. Reprenant l'idée d'une source extérieure à la voix, Felice en donne une description très matérielle qui mêle en un réseau d'images assez dense la voix et le souffle («breath»):

Felice. Piero, stay, for I descry a fume

Creeping from out the bosom of the deep,

The breath of darkness, fatal when 'tis whist

In greatness' stomach. This same smoke, called pride,

Take heed, she'll lift thee to improvidence

And break thy neck from steep security $[\ldots]$

Heaves up their heart with swelling, puffed conceit

Till their souls burst with venomed arrogance.

C'est bien une voix que cette «breath of darkness», et elle traverse Piero tout entier («stomach, heart, soul») pour lui donner souffle : «heaves up their heart», dit Felice, reprenant le même verbe utilisé par Piero («heavèd up on wings of fair success»). Le commentaire de Felice est donc une sorte de déconstruction de la voix ducale, dont il accepte l'origine extérieure, mais cette source n'est plus qu'une épaisse fumée nauséabonde. Tout ce passage prend davantage de relief si l'on considère la physique des tremblements de terre. Selon la théorie antique la plus répandue (reprise à l'époque élisabéthaine par Spenser dans A Pleasant and Pithy Familiar Discourse of the Eathquake in April Last), les tremblements 
de terre sont occasionnés par un «pneuma» enfermé dans la croûte terrestre : lorsque la pression est trop violente, l'air perce la surface de la terre et se répand au dehors. Mais quel est donc cet air qui s'échappe?

Dans ses Questions d'histoire naturelle, Sénèque propose entre autres, selon les autorités qu'il passe en revue, deux interprétations opposées. Ce «pneuma» peut être nourriture des corps célestes : «omnes hae stellae [...] alimentum ex terreno trahunt et inter se partiuntur nec ullo alio scilicet quam halitu terrarum sustinentur» ${ }^{3}$. Pourtant, selon une autre analyse, ces souffles longtemps emprisonnés dans les profondeurs arrivent à la surface viciés; ils souillent et infectent notre atmosphère, apportant toutes sortes de maladies : «aer ipse, qui vel terrarum culpa vel pigritia et aeterna nocte torpescit, gravis haurientibus est $[\ldots .]. »{ }^{4}$. Nourriture céleste ou pestilence ? Il semble que dans cette première scène d'Antonio and Mellida, nos deux personnages choisissent des interprétations différentes : pour Piero, qui semble ne pas comprendre l'attaque de Felice, c'est une nourriture bienfaisante. N'est-il pas déjà monté dans les cieux à la place des dieux, comme il le déclare superbement à son adversaire : «dimitto superos, summa votorum attigi»? Dans le passage dont est tirée cette citation du Thyeste de Sénèque, Atrée se déclare d'ailleurs «aequalis astris»; tout ce réseau d'échos et de citations indique que Piero se range du côté de la première interprétation: l'air qui monte de la terre est l'aliment des dieux. Selon Felice, au contraire, cette «breath of darkness» n'est que fumée pestilentielle qui envahit Piero et lui donne la voix de l'orgueil.

Dans la première scène d'Antonio's Revenge, la deuxième partie du dyptique, Marston fait émerger de l'échange entre Piero et son serviteur Strozzo un autre discours sur l'impérialisme vocal de Piero. Tout ce début de scène fonctionne comme une réécriture négative du livre de la Genèse : la nature est plongée dans une torpeur propice au recommencement, mais le souffle de Dieu ne plane pas à la surface des eaux :

'Tis yet dead night, yet all the earth is clutched In the dull leaden hand of snoring sleep;

No breath disturbs the quiet of the air, No spirit moves upon the breath of earth, Save howling dogs, night-crows, and screeching owls, Save meagre ghosts, Piero and black thoughts. 
Le souffle divin est remplacé par des voix stridentes et la présence de Piero qui cherche à occuper l'espace et la parole. «One, two», une cloche sonne les deux crimes comme la voix de Dieu ordonnait les éléments, mais la création est remplacée par une destruction, en particulier une destruction de la voix des autres, c'est-à-dire de leur souffle de vie. Lorsqu'il commente le meurtre d'Andrugio, Piero dit en une expression saisissante : «this brain hath choked / The organ of his breast» (I.1.14-5). Jouant sur la polysémie de «organ» (organe du corps mais aussi orgue comme instrument de musique et la voix humaine), Piero conçoit l'élimination d'un adversaire politique comme étouffement de sa voix : il s'agit de faire taire tous ses concurrents pour que le ciel admire son jeu sans partage : "You horrid scouts that sentinel swart night, / Give loud applause from your large palms» (I.1.19-20) et plus loin, «Hell, Night, give loud applause to my hypocrisy» (I.1.30-1).

Ce n'est déjà plus le même Piero que dans Antonio and Mellida. Au lieu de jouer avec la dissociation de sa voix, il semble maintenant prisonnier de cette force qui le traverse : «I can scarce coop triumphing vengeance up / From bursting forth in braggart passion» (I.1.11-2). Plus il parle, empêchant même Strozzo de placer davantage que des monosyllabes, plus sa voix engloutit le réel; et pourtant, la rhétorique de Piero ne parvient pas à naître, c'est une voix qui est incapable de parler. Une expression qui fait oxymore nous renseigne sur cette impuissance lorsque Piero, désirant accoucher de cette voix qui le travaille, dit à son serviteur : «Stroke not the head / Of infant speech till it be fully born» (I.1.39-40). Tout laniniste aura reconnu la puissante opposition «infant speech»: la rhétorique est condamnée à être «infans», privée de parole, de sorte que Piero, après avoir éliminé les voix de ses opposants, est incapable d'affirmer la sienne propre.

De façon très intéressante, l'exécution de Piero à la fin d'Antonio's Revenge est d'abord conçue comme élimination vocale : le tyran est puni par sa propre méthode. Les conspirateurs commencent par lui arracher la langue : «We'll spoil your oratory out with his tongue» (V.3.65). Puis ils lui présentent une assiette contenant le corps de son fils Julio ; celui qui reprenait des bribes empruntées au personnage d'Atrée subit le même sort qu'Atrée destine à Thyeste dans la pièce de Sénèque. On retrouve cette relation chère aux psychanalystes entre la gorge, organe de la voix, et l'engendrement : «Look, look here; / Here lies a dish to feast thy father's gorge; / There's flesh and blood which I am sure thou lov'st» (V.3.79-81). «Thy father's gorge», c'est-à-dire non la gorge de 
ton père mais ta gorge de père, comme s'il avait mis au monde son fils par la voix et devait comme punition le dévorer à nouveau. Dans la scène qui suit, les conspirateurs organisent une sorte de première mort symbolique, celle de Piero acteur, commentant ses gestes comme s'il avait émis une voix. Enfin la sanction finale reprend le mot utilisé auparavant par Piero («choked») et la même structure de phrase; nous n'avons pas «this brain hath choked the organ of thy breast» mais «the hand of heaven chokes / The throat of murder» (V.3.108-9), une formulation qui fait parfaitement écho à la première scène : Piero, qui recherchait l'approbation des mains immenses de la nuit est ici puni par la main du ciel; son souffle est étouffé comme celui d'Andrugio. On remarquera en outre l'importance de l'article défini : non pas «thy throat of murder» sur le modèle de «thy father's gorge» mais «the throat». Le crime est donc défini comme une force extérieure que l'on atteint à travers la personne du tyran qui n'en est que le vecteur.

\section{Fantaisies de l'évanouissement}

Pour Antonio, 1'opposant principal de Piero dans les deux pièces de ce dyptique, la voix est une constante agonie, une force violente dont il est persuadé qu'elle se détachera bientôt de son enveloppe corporelle pour mener une existence libre et indépendante. Antonio parle comme si chacun de ses mots était le dernier et semble caractérisé tout au long de la pièce par une puissante fantaisie de l'évanouissement, comme si son souffle devait le quitter. Il suffit de prendre le tout début d'Antonio and Mellida :

Heart, wilt not break? And thou abhorrèd life, Wilt thou still breathe in my enragèd blood? Veins, sinews, arteries, why crack ye not, Burst and divulsed with anguish of my grief ? Can man by no means creep out of himself And leave the slough of viperous grief behind?

«Breath» s'entend ici encore aux deux sens du terme : le souffle de vie qui s'exhale du sang, mais aussi la voix qui risque de faire périr le corps à force de chagrin. L'exemple le plus spectaculaire d'une dissociation entre le souffle et le corps se trouve au début de l'acte IV d'Antonio and Mellida. Dans un discours complexe, Antonio décrit une spectaculaire scission de son être : l'esprit et le corps ici 
mènent une existence séparée. Dans une pose réflexive typiquement marstonienne, le personnage s'apostrophe lui-même, comme un metteur en scène qui se donnerait ses propres directives :

Stop, stop Antonio ! Stay, Antonio !

Vain breath, vain breath; Antonio is lost.

He cannot find himself, not seize himself.

(IV.1.1-3)

Ce que nous entendons est donc une voix vide («vain breath», une expression qui rappelle l'Ecclésiaste, mêlant l'hébreu «hébel», souffle ou buée, et la traduction latine traditionnelle par «vanitas»), une non-voix. Étrange paradoxe que celui-ci : un acteur parle pour dire qu'il n'a pas de voix! Son vrai souffle, sa vraie voix sont ailleurs, incapables de parler parce que privés de corps. La dissociation est complète: le corps est sans voix en l'absence de l'esprit qui l'animait ; ce même esprit, sans le soutien du corps, ne peut devenir émission vocale :

But poor, poor soul, wanting apt instruments

To speak or see, stands dumb and blind.

L'esprit d'Antonio plane à la cour de Venise, tandis que sur scène, devant les spectateurs, il ne reste plus qu'une enveloppe («lodge», «trunk») désertée par l'esprit, sans souffle propre sinon une trace, ce qui reste de voix lorsque l'esprit quitte le corps, un parfum. Nous retrouvons ici la richesse de sens de «breath», qui signifie à la fois souffle, voix et odeur : «This little breath of sweet / Holds still a faint perfume of his sweet guest» (15-6). Quelle est donc cette voix, écartelée entre l'esprit et le corps ? Comme pour illustrer aussitôt cette fantaisie de dissociation, Marston met en scène une double mort symbolique: tandis que le véritable souffle est englouti dans de sombres nuages («rolled up», un verbe qui revient souvent dans Antonio and Mellida), le corps maintenant privé de voix tombe lourdement sur les planches («clod upon clod thus fall» (27)) et ne se relèvera qu'à l'appel de son nom, dans un échange spectaculaire entre Antonio et son père, une sorte de scène d'écho qui manipule jusqu'au vertige les conceptions métadramatiques sur l'identité de l'acteur : 
And. My dear sweet boy, my dear Antonio.

Ant Antonio?

And Ay, echo, ay, I mean Antonio.

Ant. Antonio ? Who means Antonio ?

And Where art? What art? Knows't thou Antonio ?

Ant. Yes.

And Lives he?

Ant.

And.

No.

Ant.

Where lies he dead?

Anil.

Here.

Ant. Here,

Where?

(IV.1 89-95)

dit Antonio en se relevant du sol, se déclarant mort d'une voix bien ferme et posée. Tous les jeux de mots possibles sur l'identité (le «ay» qui veut dire oui devenant «I», pronom personnel, etc.) et le statut de l'acteur comme ombre ou esprit se retrouvent dans ce passage qui mériterait une étude plus approfondie.

Ce désir de s'anéantir corporellement, de devenir pur souffle, pure voix se retrouve avec plus d'insistance encore dans la deuxième partie, Antonio's Revenge. Prenant l'habit du fou pour se déguiser à la cour comme son contemporain Hamlet, il souffle des bulles à la face de tout le monde, distribuant un commentaire classique sur la légèreté et l'insignifiance de cette vie artificielle: «Puff, hold, world! Puff, hold, bubble ! [...] And now I laugh like a good fool at the breath of mine own lips» (IV.1.100-3). Pourtant, cette nature aérienne qu'il renvoie à la cour en une image déformée et parodique, il la souhaite pour lui-même ; un peu avant l'exécution de Piero, il s'exclame: «Then will I dance and whirl about the air. I Methinks I am all soul, all heart, all spirit» (V.3.47-8). Il semble même que le devoir de revanche imposé par son père le fasse participer progressivement à cette nature aérienne du fantôme qu'il aspire à retrouver. Lors d'une de leurs dernières rencontres avant que la vengeance ne s'accomplisse, Antonio semble se rapprocher de son père, partager sa nature pneumatique, se transformer en souffle pur, libéré des entraves du corps :

See, unamazed I will behold thy face,

Outstare the terror of thy grim aspect,

Daring the horrid'st object of the night.

Look how I smoke in blood, reeking the steam

Of foaming vengeance. $\mathrm{O}$ my soul's enthroned 
In the triumphant chariot of revenge, Methinks I am all air and feel no weight Of human dirt clog. This is Junio's blood, Rich music, father, this is Junio's blood.

(III.3.73-81)

Le sang versé émet une sorte de souffle musical et Antonio, dans sa fantaisie, se transforme peu à peu en exhalaison du sang («smoke», «reeking», «steam»), en fumée libérée du corps. On notera la diversité des sens de l'adjectif «rich» qui désigne à la fois la texture dense du sang et, dans un contexte spécifiquement musical, une harmonie chargée et complexe. Il est possible de percevoir dans cette belle expression un écho de Genèse, IV.10, où le sang d'Abel (toujours de l'hébreu «hébel», le souffle, la buée) crie vers Dieu depuis la terre où il repose : «The voice of thy brother's blood crieth unto me from the ground». L'allusion biblique fonctionne ici comme un contre-discours minant la phrase de l'intérieur et venant rappeler que le sang versé est celui d'un sacrifice illicite.

En dehors des deux voix que nous venons d'examiner, le dyptique de Marston contient également le personnage de Balurdo: il partage les prétentions impérialistes de Piero quant à la voix et aussi le désir manifesté par Antonio de devenir pur souffle. S'imposer par la voix, c'est bien ce qu'il cherche lui aussi, par tous les moyens, même s'il faut pour cela faire parler un tableau. A l'acte $\mathrm{V}$ scène 1 d'Antonio and Mellida, Balurdo demande au peintre de lui construire un emblème de sa propre voix; après le son olfactif, voici le son visuel... et olfactif aussi sans doute puisqu'il s'agit d'un rot :

\footnotetext{
Balurdo. God's nigs, now I remember me, I ha' the rarest device in my head that ever breathed. Can you paint me a drivelling, reeling song, and let the word be "Uh" ?

Fainter. A belch?

Balurdo. O no, no, no "Uh". Paint me "Uh" or nothing.
}

Dans la deuxième scène de l'acte $\mathrm{V}$, où Piero organise un concours de chant entre deux jeunes pages, Balurdo rivalise avec le tyran en demandant à tout le monde de regarder son souffle s'échapper d'une harpe jouée par quelqu'un d'autre : «Hold, hold, hold! Are ye 
blind ? Could you not see my voice coming from the harp ?» (V.2.1920). Lui qui déclare solennellement dans Antonio's Revenge «I was made knight only for my voice» (III.3.44-5) dit comme Antonio, mais dans une version déformée et grotesque : «I could belch rarely, for I am all wind» (V.2.10-1), ce qui peut se lire comme un écho bouffon de la phrase d'Antonio «Methinks I am all air». Comme son intervention dans la première scène d'Antonio and Mellida pouvait le laisser penser, Balurdo occupe dans l'économie des voix un rôle de déformation, une contrepartie ridicule des analyses sérieuses conduites par Marston sur les autres personnages. A la fin de l'expérience dramatique, le bilan est un affrontement de discours et de voix qui finissent par se détruire mutuellement à force de combats.

Le problème principal dans ces pièces expérimentales de Marston est ce que l'on pourrait appeler l'incarnation de la voix, c'est-à-dire le passage du souffle dans un corps qui produit l'émission vocale. Que l'on manipule cette dissociation dans le sens d'une affirmation machiavélique comme Piero dans Antonio and Mellida ou que l'on en souffre comme Antonio ou le même Piero dans Antonio's Revenge, la voix est toujours présentée comme extérieure, faisant violence au corps qui la transmet. Au delà des réflexions sur le statut de l'acteur (la pièce commence par cette fameuse scène où les acteurs sous cape discutent des rôles qu'ils vont interpréter), Marston nous livre une thématisation de la voix politique sous deux de ses aspects, celle du tyran et celle de l'opposition. Nous pouvons ouvrir cette brève réflexion sur la prochaine pièce politique de Marston (The Malcontent), où le personnage de Malevole, lui-même décrit comme souffle, comme vent ( He is as free as air, he blows over every man» (I.1.39)) possèdera cependant une identité problématique et une liberté de parole toujours menacée par le jeu de l'acteur, comme le révèle l'expression paradoxale : «Play I well the free-breathed discontent ?» (1.1.234) — «free-breathed», certes, mais ce n'est encore que parole d'acteur, parole liée, comme possédée par un autre que soi. 


\section{NOT E S}

1 «Antonio's Revenge first of all dramatises the way that dislocation in the world generates dislocation in consciousness. "Grief" and what it stands for in terms of estrangement, alienation and disorientation threatens not just the individual's capacity to survive the world, but his very identity within it » (Jonathan Dollimore, Radical Tragedy, The Harvester Press, 1984, chapter 2, p. 34).

2 Toutes nos citations de Marston seront tirées de l'édition réalisée par Keith Sturgess, The Malcontent and Other Plays, Oxford University Press, 1997.

3 «Toutes ces étoiles [...] tirent de la terre une alimentation qu'ils se partagent entre eux et n'ont à coup sûr d'autre subsistance que les exhalaisons du sol» (Sénèque, Questions naturelles, chapitre VI «de terrae motu», XVI-2, traduction de Paul Oltramare, Les Belles Lettres, 1929, p. 270).

${ }^{4}$ lbid., section XXVII-2, p. 284. 